\title{
Assessment of age-related skin changes using hyperspectral polarization imaging
}

Viktor Dremin, Alexander Bykov, Zbignevs Marcinkevics, Andris Grabovskis, Evgeny Zherebtsov, et al.

Viktor Dremin, Alexander Bykov, Zbignevs Marcinkevics, Andris Grabovskis, Evgeny Zherebtsov, Alexey Popov, Igor Meglinski, "Assessment of agerelated skin changes using hyperspectral polarization imaging," Proc. SPIE 11079, Medical Laser Applications and Laser-Tissue Interactions IX, 1107918 (22 July 2019); doi: 10.1117/12.2526359

SPIE. Event: European Conferences on Biomedical Optics, 2019, Munich, Germany 


\title{
Assessment of Age-related Skin Changes Using Hyperspectral Polarization Imaging
}

\author{
Viktor Dremin ${ }^{\mathrm{a}, \mathrm{b}}$, Alexander Bykov ${ }^{\mathrm{a}}$, Zbignevs Marcinkevics ${ }^{\mathrm{c}}$, Andris Grabovskis ${ }^{\mathrm{d}}$, \\ Evgeny Zherebtsov ${ }^{\mathrm{a}, \mathrm{b}}$, Alexey Popov ${ }^{\mathrm{a}}$, and Igor Meglinski ${ }^{\mathrm{a}}$ \\ ${ }^{a}$ Optoelectronics and Measurement Techniques Unit, University of Oulu, Erkki \\ Koiso-Kanttilankatu 3, 390014, Oulu, Finland \\ ${ }^{\mathrm{b}}$ Research \& Development Center of Biomedical Photonics, Orel State University, \\ Komsomolskaya Str. 95, 302026, Orel, Russia \\ ${ }^{c}$ Department of Human and Animal Physiology, University of Latvia, Jelgavas str. 1, LV-1004, \\ Riga, Latvia \\ ${ }^{d}$ Biophotonics Laboratory of Institute of Atomic Physics and Spectroscopy, University of \\ Latvia, Jelgavas str. 3, LV-1004, Riga, Latvia
}

\begin{abstract}
We report a development of hyperspectral polarization imaging approach for assessment of age-related skin changes. The degree of residual polarization is used as a quantitative marker of the age-related pathological malformations. The developed approach in frame of express analysis of the degree of residual polarization in each pixel of the image are shown.
\end{abstract}

Keywords: hyperspectral imaging, polarization, collagen, advanced glycation end-products, diabetes mellitus

\section{INTRODUCTION}

The accompanying structural and functional changes of collagen during tissue glycation make a significant contribution to the development of age-related pathologies associated with diabetes mellitus (DM), affecting the heart and blood vessels, eyes, kidneys, nerves, causing a number of complications that lead to a high risk of disability and a threat to life. ${ }^{1}$ According to the International Diabetes Federation (IDF), the problem of early diagnosis and monitoring the effectiveness of DM treatment is one of the most acute in modern healthcare. DM is one of the ten deadliest diseases in the world, with a yearly mortality rate over 1M. In 2017 there were $425 \mathrm{M}$ patients with DM in the world, and by 2045 this figure will increase to $629 \mathrm{M} .{ }^{1}$ Recent studies have noted that timely diagnosis and treatment, including increasing patient control, reduces complications and makes it possible to reverse their development if detected in the early preclinical stages.

In recent years, it has been established that developing DM, as well as human aging leads to an increase in the level of protein glycation. This is accompanied by an increase in the so-called advanced glycation endproducts (AGEs), ${ }^{2}$ which affect the properties of collagen and other structural proteins of the capillary membrane and skin. By monitoring collagen structures, we are able to evaluate skin status and inspect age-related skin changes. These changes can be recorded, for example, using fluorescence measurements. ${ }^{3}$ The diffuse reflected light penetrates the skin and depolarizes by the birefringent skin collagen fibers. Interaction of polarized light with human skin was studied previously see, for example, Refs. ${ }^{4,5}$ Prediction of diabetic ulcers can also be based on detection of changes in skin structure and formation of collagen scarring, which can be detected via polarization imaging even when fibrosis is invisible by naked eye.

The aim of current study is to introduce and test a hyperspectral polarization imaging device and the method for monitoring age-related human skin changes in vivo, as well as the sensitivity of the method to the recognition of skin scars.

Send correspondence to Viktor Dremin

E-mail: viktor.dremin@oulu.fi,

Telephone: +358414706340

Medical Laser Applications and Laser-Tissue Interactions IX, edited by Lothar D. Lilge, Carsten M. Philipp, Proc. of SPIE Vol. 11079, $1107918 \cdot$ @ 2019 SPIE · CCC code: 1605-7422/19/\$21 · doi: 10.1117/12.2526359 


\section{MATERIAL AND METHODS}

We use a modified version of the hyperspectral imaging system based on a Fabry-Perot interferometer. The detailed description of hyperspectral system is presented in Ref. ${ }^{6,7}$ In the present study, we adapt this system to measure the degree of residual polarization of radiation reflected from a biological tissue. The system was equipped with a rotating linear polarizer, which can be placed either parallel or perpendicular to the initial polarization of the light source. Two hyperspectral images were acquired $I_{c o}$ when the analyzer was parallel to the illumination and $I_{\text {cross }}$ when the analyzer was perpendicular to the illumination. The degree of residual polarization is defined as:

$$
D O P=\frac{I_{c o}-I_{\text {cross }}}{I_{c o}+I_{\text {cross }}}
$$

To obtain the residual polarization spectra, the hyperspectral cube was normalized to the images of the reflectance standard. 32 healthy volunteers (9 males and 23 females, aged 22-76 years) were recruited to participate in the experiment. In total, over 100 hyperspectral images were recorded. Hyperspectral images of skin in coand cross-polarization were recorded on the palm dorsal surface, as well as in the scar areas. Then, in accordance with Eq. 1, the degree of residual polarization was counted.

\section{RESULTS AND DISCUSSION}

Fig. 1a shows the averaged spectra of residual polarization for two volunteers groups: under 40 years and over 40 years. Hyperspectral measurements make it possible to calculate the integral parameter of residual polarization over the entire wavelength range as an area under the residual polarization spectrum (total $D O P$ ). A statistically significant increase of this parameter in elderly volunteers can be observed (Fig. 1b). Fig. 1c shows the significant relationship between total $D O P$ and age $(r=0.89, p<0.001)$.
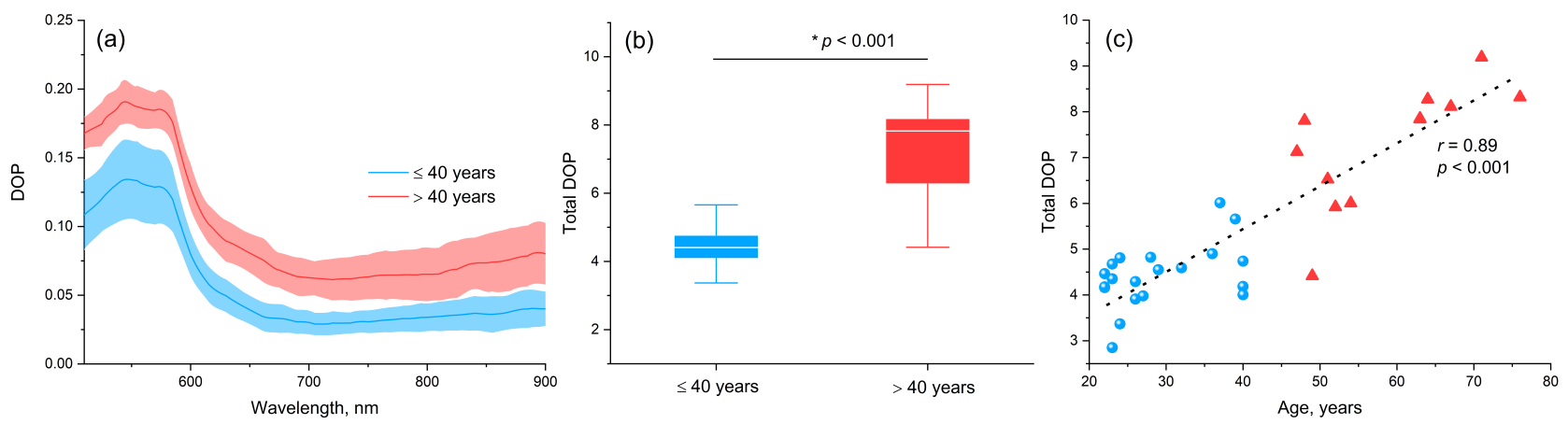

Figure 1. (a) Residual polarization spectra for two age groups: up to 40 years and after 40 years; (b) comparison of total $D O P$ among two age groups; (c) relationship between total DOP and age (blue circle - under 40 years; red triangle over 40 years). A MannWhitney $U$-test was used to identify differences between the two groups.
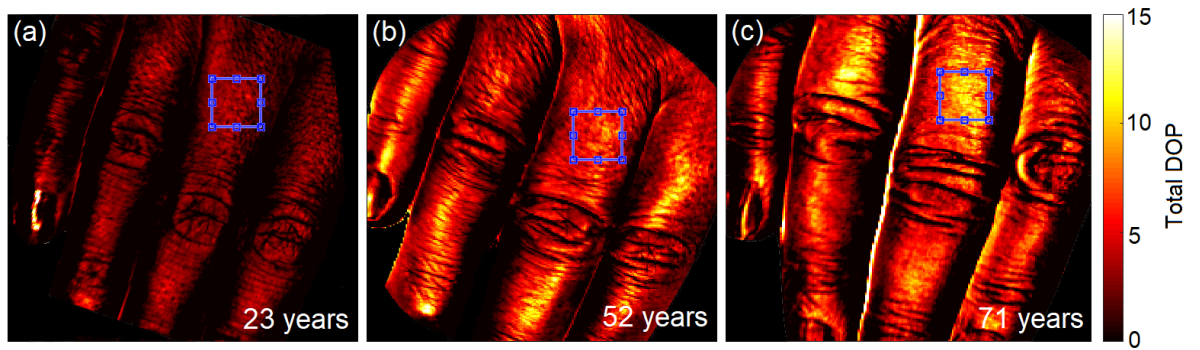

Figure 2. An example of the total DOP images: (a) 23 years; (b) 52 years; (c) 71 years. ROI indicates the averaging area to obtain the residual polarization spectra. 
Fig. 2 shows examples of the total $D O P$ images for three female volunteers of different ages. The above dependence of the total $D O P$ on age is observed.

Additionally, the scar was studied to assess the possibilities of the presented method for monitoring collagen changes in biological tissues. Fig. 3a,b show the images of the finger scar obtained for co- and cross-polarized light detection, respectively, in the spectral band of hemoglobin absorption $(580 \mathrm{~nm})$. The choice of this wavelength is associated with better visualization of the upper affected skin layers. The images in co- and cross-polarization show hard to distinguish regions of scar. However, the degree of residual polarization image (Fig. 3c) shows this region as a more contrasting signal relative to normal skin. Approach with calculation of total DOP can significantly improve visualization of alterations in collagen structure and the contrast of the image (more than $10 \%$ ), as shown by the arrow in Fig. 3d.

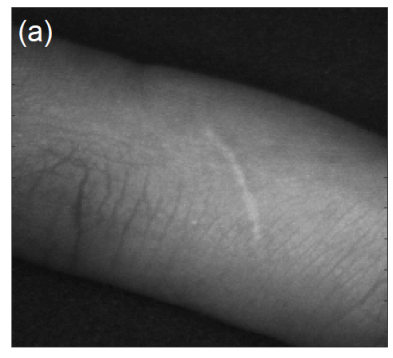

Figure 3. Images of finger scar: polarization spectrum.
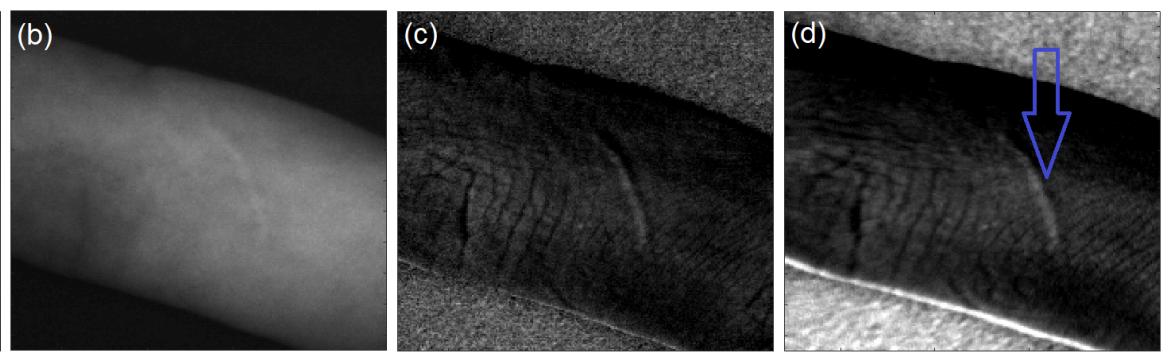

We assume that the shown differences, characterized by age-related collagen changes, can form the basis for the diagnosis of DM complications. As already mentioned, AGEs contribute to the mechanisms responsible for the development of complications in diabetes. Formation of intracellular AGEs promotes violation in protein function, with their accumulation acting as an objective marker for the tissues glycation. Changes in AGE content reflect long-term processes in the diabetic skin. ${ }^{2}$ Considering the long molecular lifetime of collagen and the stability of AGEs, it is possible to use degree of residual polarization as a measure of the total impact of hyperglycemia throughout the course of life.

It should be borne in mind that the blood content in the biotissue, epidermis thickness and some other factors may affect the residual polarization spectra. Thus, hyperspectral polarization imaging makes it possible to selectively monitor the change in the concentration of different chromophores in biotissue.

\section{CONCLUSION}

We conclude that hyperspectral polarization imaging shows promise for in vivo noninvasive real-time assessment of age-related skin changes. Availability of new diagnostic tool in remote areas have a high potential to increase the coverage of population screening and improve healthcare and quality of life. The results of the work can form the basis for the development of innovative screening medicine and self-management medicine. Thus, the development of new optical diagnostic methods has potential to find promising application in diagnosing diabetes disorders.

\section{ACKNOWLEDGMENTS}

Authors acknowledge the support of the Academy of Finland (grants: 290596, 314369). The work was also funded by RFBR according to the research project 18-02-00669 (processing of hyperspectral data).

\section{REFERENCES}

[1] [IDF Diabetes Atlas. 8th edition], International Diabetes Federation, Brussels, Belgium (2017).

[2] Gkogkolou, P. and Bohm, M., "Advanced glycation end products: key players in skin aging?," DermatoEndocrinology 4(3), 259-270 (2012). 
[3] Dremin, V., Zherebtsov, E., Sidorov, V., Krupatkin, A., Makovik, I., Zherebtsova, A., Zharkikh, E., Potapova, E., Dunaev, A., Doronin, A., Bykov, A., Rafailov, I., Litvinova, K., Sokolovski, S., and Rafailov, E., "Multimodal optical measurement for study of lower limb tissue viability in patients with diabetes mellitus," $J$. Biomed. Opt. 22(8), 085003 (2017).

[4] Tuchin, V. V., "Polarized light interaction with tissues," J. Biomed. Opt. 21(7), 071114 (2016).

[5] Kunnen, B., Macdonald, C., Doronin, A., Jacques, S., Eccles, M., and Meglinski, I., "Application of circularly polarized light for non-invasive diagnosis of cancerous tissues and turbid tissue-like scattering media," $J$. Biophoton. 8(4), 317-323 (2015).

[6] Zherebtsov, E., Popov, A., Doronin, A., Meglinski, I., and Bykov, A., "Hyperspectral system for imaging of skin chromophores and blood oxygenation," Proc. SPIE 10412, 104120G (2017).

[7] Zherebtsov, E., Dremin, V., Popov, A., Doronin, A., Kurakina, D., Kirillin, M., Meglinski, I., and Bykov, A., "Hyperspectral imaging of human skin aided by artificial neural networks," Biomed. Opt. Express 10(7), 3545-3559 (2019). 